\title{
TEXTURES AND BARRIER PROPERTIES OF ETHYLENE-VINYLALCOHOL COPOLYMER FILMS
}

\author{
TAKASHI ISHIDA* , TAKAYUKI SOUTOME** \\ and KAZUYOSHI SEKINE**
}

* Plastics Laboratory, Nippon Petrochemicals Co.Ltd. 2-3-2, Yako, Kawasaki-ku, Kawasaki, 210 Japan

** Faculty of Engineering, Yokohama Nat. University, 156, Tokiwadai, Hodogaya-ku, Yokohama, 240 Japan

\section{Abstract}

The effect of textures produced by various types of deformation processing on gas barrier property of copolymer film has been studied in the case of ethylene-vinylalcohol (EVOH) resin which is known as a semicrystalline polymer with low gas permeability.

The heavily cold rolling and cold stretching give rise to highly oriented molecular structures and strong crystallographic textures, which reduce gas permeability of the film at high humidity. It was found, in particular, that the increased tri-axial orientation type texture and resulting molecular structure changes due to the plane strain compression type deformation, such as rolling, improved greatly the barrier property of the EVOH film.

\section{Introduction}

The ethylene-vinylalcohol copolymer (EVOH), which is a semicrystalline polymer, has a good barrier property against gaseous permeation and hence is used as a flexible packaging film of food products. The barrier properties of EVOH film depend on the molecular structures, especially, on crystallinity and on the orientation, which are strongly affected by heat treatment and deformation processing. The importance of crystallinity to gas barrier properties of polymers has been examined and recognized.(1)

However, the study on the EVOH has been done very little from the standpoint of crystallographic textures.(2) In this paper, the effects of various types of deformation processing and their resulting textures on the barrier property of EVOH films have 
been studied in order to improve the performance as the packaging film.

\section{Experimental}

The material used in this study was a EVOH (EVALF101 of Kularay Co. Ltd., Japan) having ethylene content of $32 \mathrm{~mol} .8$. The EVOH crystal containing $32 \%$ ethylene is considered to be monoclinic. (3)

The EVOH resin was processed into a coextruded multilayer film, which was laminated with lowdensity polyethylene on both sides by flat-film extrusion. Polyethylene layers on both sides eliminate the effect of the roll contact on the central layer of EVOH during deformation processing.

The test samples were made by uni- and bi-axial stretching and uni-directional rolling with various draw ratios at the temperature of $90^{\circ} \mathrm{C}$. While conducting the deformation processing, the film was preheated by contact with a series of preheating rolls to facilitate processing (contact length $1000 \mathrm{~mm}$, temperature $70^{\circ} \mathrm{C}$ ). Some of samples were subsequently heat-treated at the temperature of 120,140 and $160^{\circ} \mathrm{C}$ for 1 hour.

The textures of samples were determined by drawing (110) pole figures using the Decker's transmission and the Schultz's reflection methods.

The determination of $\mathrm{O}_{2}$ gas permeation constant of the samples has been carried out by oxygen permeability tester (OX-TRAN manufactured by Modern Controls, Inc.). Gas permeation can be expressed as follows: $\quad m=P \quad(\Delta p / L)$

where $\mathrm{m}$ is the permeation rate per unit area of the film, $P$ is the permeation constant, $\Delta p$ is the partial pressure difference of oxygen at both film surfaces, and $\mathrm{L}$ is the film thickness. In the experiment, $\Delta p$ was set and $\mathrm{m}$ and $\mathrm{L}$ were measured to obtain the permeation constant $P$. The gas permeation constant varies according to the temperature and the humidity. In this experiment, $\mathrm{P}$ were determined at $30^{\circ} \mathrm{C}$ under very high humidity (about $100 \% \mathrm{RH}$ ) .

The degrees of crystallinity of the samples were estimated by the measurements of their densities.

3. Results

Fig.1 shows the results of $\mathrm{O}_{2}$ gas permeation 
measurement for the samples subjected to various types of processing. It is clear that in the cases of uni-axial stretching or uni-directional rolling, the degree of $\mathrm{O}_{2}$ gas permeation is significantly reduced. In particular, the effect of uni-directional rolling is remarkable in that the $\mathrm{O}_{2}$ gas permeation constant decreases to $1 / 10$ of the undeformed sample. The results of $\mathrm{O}_{2}$ gas permeation measurement after heat treatment are shown in Fig.2. All of the deformed samples exhibit low gas permeation constants after the heat treatment. This improvement was remarkable in the uni-directional rolling samples subsequently subjected to heat treatment. It was also found that the most excellent barrier property can be obtained by combining the process of uni-directional rolling and subsequent heat treatment.

The (110) pole figure of undeformed EVOH sample exhibits a random crystallite orientation distribution.

The (110) pole figure for the sample uni-axially stretched to the draw ratio of 3 is given in Fig. 3 . This texture is considered to be not so strong. A tendency can be seen whereby (110) poles accumulate on the equator of the pole figure, that is, the [001] axis of EVOH crystallites (the molecular chain axis) aligns parallel to the stretching direction of the film (uni-axial orientation).

In the bi-axially stretched sample (Fig.4), a crystallite texture, in which (110) poles are densely accumulated at the center of the pole figure, is formed (bi-axial orientation).

On the other hand, the sample uni-directionally rolled to the draw ratio of 3 (Fig.5) has the texture, in which the (110) poles accumulate on the equator, in particular at the center of the pole figure. This texture means that the molecular chain axis aligns parallel to the rolling direction as in the case of uni-axial stretching, furthermore, there is a tendency for the (110) plane aligns parallel to the film surface (tri-axial orientation). This texture formed by the uni-directionally rolling are very strong .

Several results of the density measurement are shown in Fig.6. Whereas the density is significantly increased by the heat treatment, there are no major differences in the density among three samples subjected to three types of processing. This fact 
means there are no significant differences of degree of crystallinity among these samples.

4. Discussion (4-5)

The experimental results suggest that the increased tri-axial orientation and its resulting morphological changes of the molecular structure achieved by uni-directional rolling (plane compressive strain type deformation) improve the barrier property of EVOH films the most effectively. This is considered to be caused by that all crystallites surfaces facing surrounding regions are fixed in the tri-axial orientation, so that the crystallites can contact with each other smoothly. Therefore, when compared with the other orientations, in which the boundaries of the crystal surfaces contact imperfectly, the tri-axial orientation provides an excellent gas barrier property. Besides, the tri-axial orientation is also attributable to the rapid progress of crystallization due to the smooth crystallites contacts, while imperfect contacts of the crystallites hinder the crystallization.

\section{Conclusions}

It can be concluded that the crystallographic textures of the EVOH films can significantly affects the gas barrier property, especially, textures with the tri-axial orientations formed by uni-directional rolling provides the excellent gas barrier property.

It was also found that the most excellent barrier property can be obtained by combining the process of uni-directional rolling and subsequent heat treatment. The possible explanation for this relationship between the textures and the barrier properties has been discussed in the preceding section.

\section{References}

1)M.Salame, J.Plastic Film \& Sheeting,2,321 (1986)

2 ) J.D.Culter,J.Plastic Film \& Sheeting,1,215 (1985)

3 ) T.Matsumoto, K. Nakamae, N.Kogoshi, M. Kawazoe, H.Oka, Kobunshi Kagaku,28,610 (1971)

4 ) T.Katsula, Kobunshi Ronbunshu, 37,581 (1980)

5)D.C.Sun, E.M.Berg, and J.H.Magill, Polym.Eng.Sci., $30,635(1990)$ 


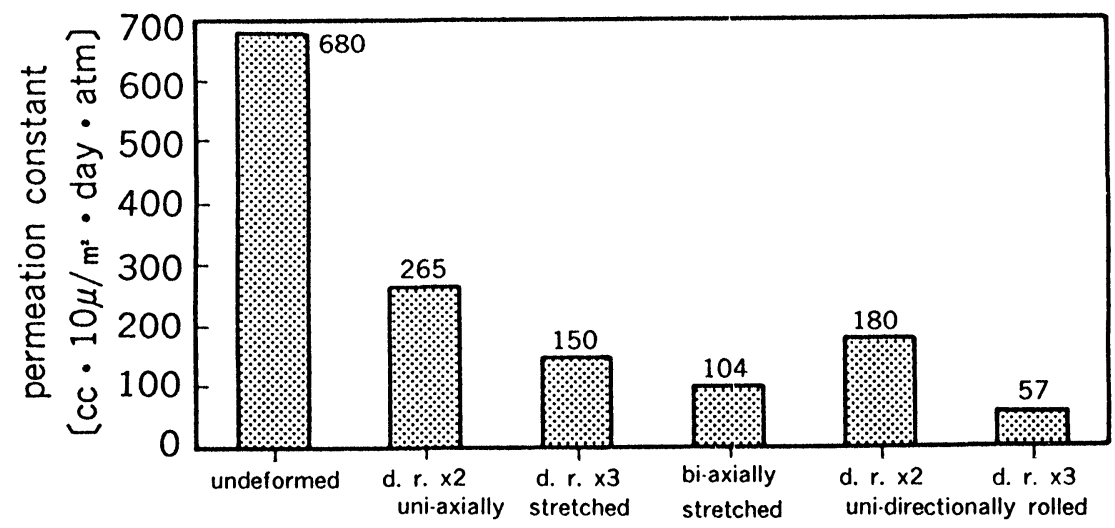

Fig. $1 \mathrm{O}_{2}$ permeation constants of EVOH subjected to various types of deformation (at $30^{\circ} \mathrm{C}$ under about $100 \% \mathrm{RH}$ )
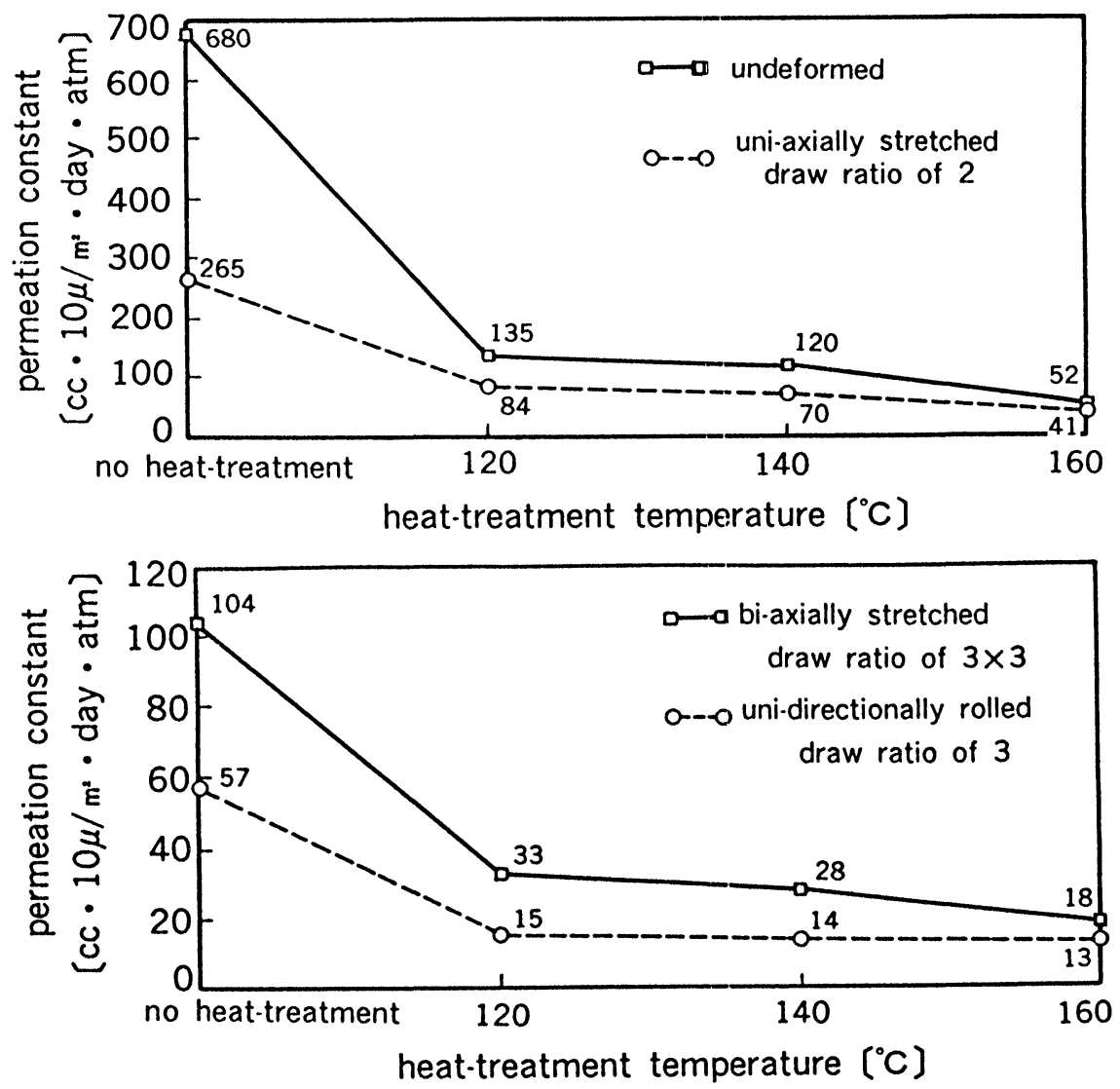

Fig. 2 changes of $\mathrm{O}_{2}$ permeation constants as a funtion of heat-treatment temprature (at $30^{\circ} \mathrm{C}$ under about $100 \% \mathrm{RH}$ ) 


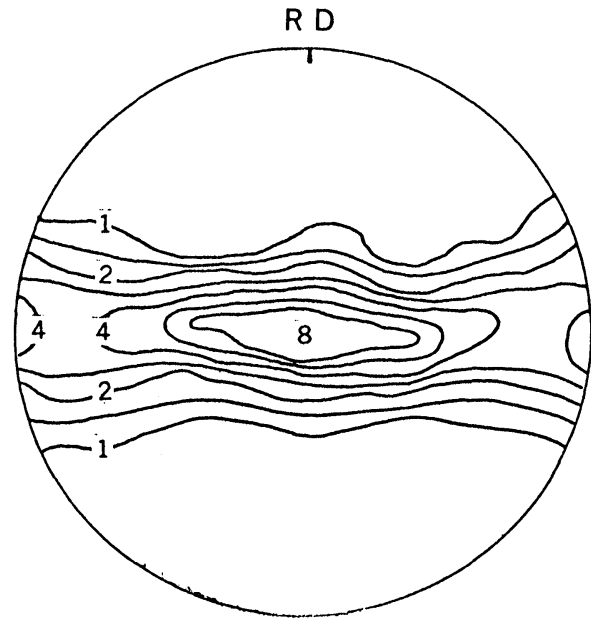

Fig. 3

(110) pole figure of EVOH uni-axially stretched at draw ratio of 3

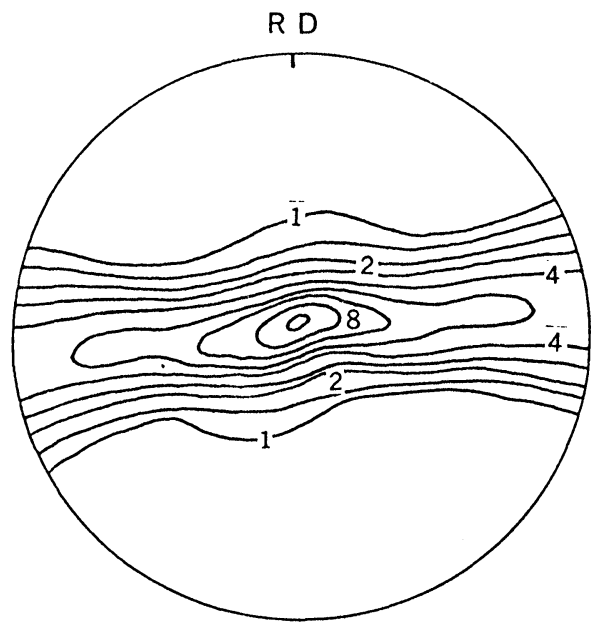

Fig. 5

(110) pole figure of $\mathrm{EVOH}$ uni-directionlly rolled at draw ratio of 3

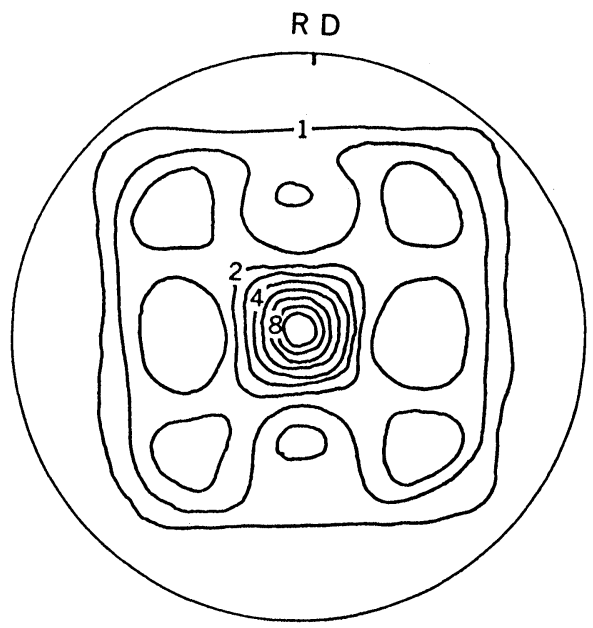

Fig. 4

(110) pole figure of EVOH bi-axially stretched at draw ratio of $3 \times 3$

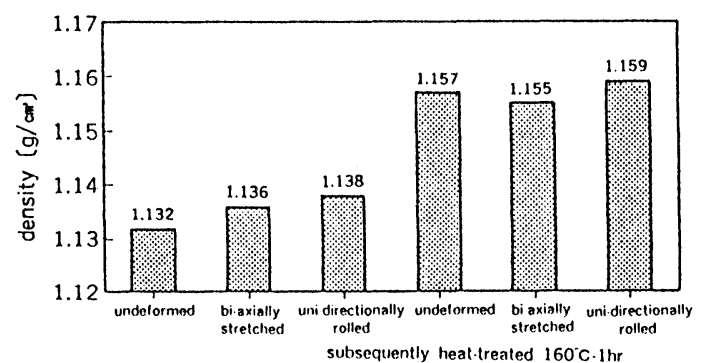

Fig. 6 densities of samples subjected to deformation and/or heat treatment 